2SB1040 溶液中 ATP 加水分解の自由エネルギー変化の分子論的解釈 Theoretical Study on the Reaction Pathway and Free Energy of ATP Hydrolysis in Solution

Takeshi Yamamoto, (Department of Chemistry, Kyoto University)

ATP (adenosine triphosphate) is known as the "molecular unit of currency" of intracellular energy transfer. It releases a relatively large amount of free energy (about $40 \mathrm{~kJ} / \mathrm{mol}$ ) upon hydrolysis in aqueous solution (ATP $+\mathrm{H}_{2} \mathrm{O}->$ ADP $+\mathrm{Pi})$. The released free energy can be utilized such that an energetically unfavorable reaction can proceed in an otherwise difficult case. For this reason, the phosphate anhydride (P-O-P) bonds in ATP are often called "high energy bonds" in literature. For the physical interpretation, many textbooks provide an intramolecular explanation that the P-O-P bond itself has a high energy and thus the cleavage of the P-O-P bond releases a large amount of energy. On the other hand, possible roles of surrounding water molecules have also been discussed in literature. For example, Wikipedia states that the strength of the $\mathrm{P}-\mathrm{O}-\mathrm{P}$ bond is less than the strength of the hydration bonds between the products $(\mathrm{ADP}+\mathrm{Pi})$ and water. This implies that water solvent provides a major driving force for the reaction. In this session, we will discuss the physical nature of the ATP hydrolysis in solution by using computer simulations based on quantum chemistry and statistical mechanics. The results indicate that bulk water molecules play the role of "suppressing" the hydrolysis reaction and they actually increase the activation barrier of hydrolysis. This result is found to be consistent with a recent experiment on phosphomonoester hydrolysis in cyclohexane that revealed $10^{12}$-fold enhancement of the reaction rate.

\section{SB1105 機能性分子およびイオンの水和の構造論とエネルギー論}

Structure and Energetics of Hydration for Functional Molecules in Solution

Nobuyuki Matubayasi, (Institute for Chemical Research, Kyoto University)

The importance of hydration is now recognized well in solution, biophysical, interfacial, and electrochemical processes. The question at present is when the hydration is important and how important it is. This question necessitates the methodological development of quantitative analysis at atomic resolution. In the present talk, we describe our theoretical/computational approach: the free-energy analysis (energetics) and the hydration-shell analysis (dynamic structure) for functional molecules such as ATP, phosphate, and proteins in water. In the free-energy analysis, we perform all-atom molecular simulation combined with the energy-representation theory of solutions and discuss the hydration free energy in connection to the solute-solvent interaction. The effect of added salt is examined for ionic hydration, and the role of attractive and repulsive interactions is elucidated for protein hydration. In the hydrationshell analysis, we present a spatial-decomposition formula which expresses the observed response of solvent to the solute insertion in terms of the integral of the local response function over the space. The contributions from the first and outer shells are separately treated, and the localization of the solute perturbation on the solvent structure and dynamics is clarified with respect to the NMR relaxation measurement and dielectric spectroscopy. In addition to the role of water, the role of nano-scale inhomogeneity produced by the presence of lipid membrane is also discussed by extending the concept of solvation.

\section{SB1350 複合体界面に見られる包まれ構造と天然変性領域の関係}

The interwinding nature of protein-protein interfaces and its implication for protein complex formation

Kei Yura ${ }^{1,2}$, Steven Hayward ${ }^{3},\left({ }^{1}\right.$ Computational Biology, Graduate School of Humanities and Sciences, Ochanomizu Univ.: ${ }^{2}$ Center for Informational Biology, Ochanomizu University: ${ }^{3}$ School of Computing Sciences, University of East Anglia)

Structural features at protein-protein interfaces can be studied to understand protein-protein interactions. Along with the accumulation of protein complex structures, we noticed that in a dataset of 45 multimeric proteins, the interface could either be described as flat against flat or protruding/interwound. In the latter, residues within one chain were surrounded by those in other chains, whereas in the former they were not. To investigate the generality of the interwound/surrounded nature of the interfaces, a simple method was developed that could distinguish between these two types with results that matched those made by human annotation. Applying this automatic method to a large dataset of protein complex structures, a significant portion of the interfaces were categorised into surrounded/interwound group. It was further found that the surrounded set had a significantly lower folding tendency using a sequence-based measure than the non-surrounded set. This suggests that before the subunits form complex structures, chains to be surrounded are relatively unstable and assume disordered structures. These disordered structures of one subunit may play a role to reel in the other subunits and help to form complex structures in a short time.
2SB1420天然変性ペプチド CARMIL によるアクチンキャッピングプロ テインの活性制御機構

Regulatory mechanism of actin capping protein by an intrinsically disordered peptide CARMIL

Shuichi Takeda ${ }^{1,2}$, Shiho Minakata ${ }^{2}$, Ryotaro Koike ${ }^{3,4}$, Ichiro Kawahata ${ }^{5}$, Akihiro Narita ${ }^{1,2,6}$ Masashi Kitazawa $^{5}$, Motonori Ota ${ }^{3,4}$, Tohru Yamakuni ${ }^{5}$, Yuichiro Maeda ${ }^{1,2,6}$, Yasushi Nitanai ${ }^{2,7}$ ( ${ }^{1}$ Structural Biology Research Center, Nagoya University: ${ }^{2}$ ERATO Actin Filament Dynamics Project, JST, clo RIKEN SPring-8 Center: ${ }^{3}$ raduate School of Information Science, Nagoya University: ${ }^{4}$ Institute for Bioinformatics Research and Development, JST: ${ }^{5}$ Department of Pharmacotherapy, Graduate School of Pharmaceutical Sciences, Tohoku University: ${ }^{6}$ Division of Biological Science, Graduate School of Science, Nagoya University: ${ }^{7}$ Structural Biophysics Laboratory, RIKEN SPring-8 Center, Hyogo)

The actin capping protein (CP) specifically binds to the barbed end of actin filaments. Although CP stably caps the barbed end in vitro (t[1/2] $30 \mathrm{~min}$ ), recent microscopic studies suggested that $\mathrm{CP}$ rapidly dissociates from the filaments in vivo $(\mathrm{t}[1 / 2] \sim 1.2 \mathrm{sec})$. CARMIL proteins (CARMIL, CD2AP, and CKIP-1) are defined by a consensus, $\sim 20$ amino acid CP-binding motif. CARMIL proteins may play a central role in the rapid $\mathrm{CP}$ turnover in living cells since they act on the barbed end-bound $\mathrm{CP}$ and facilitate its dissociation from the barbed end. However, the mechanism of this filament uncapping activity has remained unknown. Here we present the crystal structures of CP complexed with peptides derived from the CP-binding motif of CARMIL proteins. CARMIL peptides in an extended conformation bind a groove on a surface of CP distinct from its actin-binding site, indicating that the peptides inhibit $\mathrm{CP}$ in an allosteric manner. A structural comparison analysis revealed that $\mathrm{CP}$ has two stable domains that are continuously twisting relative to each other. Since the peptides bind across the two domains, we propose that CARMIL peptides attenuate the binding of CP to actin filaments by suppressing the twisting movement required for tight barbed end capping. A computational analysis using the elastic network model supports this idea. Our results that CARMIL peptides are functional with an extended backbone conformation provide new insights into the mechanism of the action of intrinsically disordered proteins.

\section{SB1450 フォスフォランバンによるカルシウムイオンポンプの柔らかい} 制御機構：シミュレーションからの知見

Molecular simulations of soft interactions between calcium pump and phospholamban

Yuji Sugita, (RIKEN Advanced Science Institute)

Phospholamban (PLN) is a 52-residue membrane protein that regulates the activity of the sarcoplasmic reticulum calcium pump in cardiac muscle. The regulation is controlled by the phosphorylation of PLN at Ser16 or Thr17: when PLN is phosphorylated, its inhibitory action is relieved, whereas unphosphorylated PLN can inhibit the activity of the pump. To investigate molecular mechanisms for the regulation, we computationally explore all possible conformations by performing REMD simulations for the cytoplasmic domain of phosphorylated (pPLN) and unphosphorylated PLN. The results showed that (i) without phosphorylation, the region from Lys3 to Ser16 takes all alpha-helical conformations; (ii) when phosphorylated, the alpha-helix is partially unwound in the C-terminal part (from Ser10 to Ala15) resulting in less extended conformations; (iii) the phosphate at Ser16 forms salt bridges with $\operatorname{Arg} 9, \operatorname{Arg} 13$, and/or $\operatorname{Arg} 14$; and (iv) the salt bridges with $\operatorname{Arg} 13$ and Arg 14 distort the alpha-helix and induce unwinding of the C-terminal part. The distortions caused by the salt bridges involving the phosphate at Ser16 substantially reduce the population of all helical conformations, which are presumably required for the binding to the calcium pump. We also performed all-atom MD simulations of pPLN and PLN in the biological membrane and found the effect of lipids on the conformational changes of PLN upon the phosphorylation is minor.

2SB1520 Staphylococcal nuclease の誘導折り畳みの機構：Folding before binding $か$ 、 Binding before folding $か$ ?

The mechanism of induced folding of Staphylococcal nuclease: Folding before binding or binding before folding?

Mikio Kataoka, (Nara Institute of Science and Technology)

Some functional proteins exist without tertiary structures under physiological condition. These proteins are called "intrinsically unstructured protein (IUP)". The ligand-induced folding would be a key to understand the functional mechanism of IUP. Two possible mechanisms for the induced folding are the folding before binding (scheme 1) and the binding before folding (scheme 2). In the scheme 1 which is the generally accepted view, a ligand can bind to the folded state only. In the scheme 2 , the binding of ligand is the required step for folding reaction. We have created some mutants of Staphylococcal nuclease (SNase) which take non-native structure under physiological condition 